\title{
Effects of butylphthalide on cognitive decline in diabetic rats
}

\author{
ZHIYAN TIAN, JINHUA WANG, YAN WANG, MIAO ZHANG and YUYING ZHOU \\ Department of Neurology, Tianjin Huanhu Hospital, Tianjin 300350, P.R. China
}

Received July 14, 2016; Accepted July 13, 2017

DOI: $10.3892 / \mathrm{mmr} .2017 .7700$

\begin{abstract}
Butylphthalide, a component extracted from seeds of Chinese celery, is an effective neuroprotective agent used for the treatment of ischemic stroke and dementia. Diabetes may cause central nervous system damage, and diabetes is closely associated with dementia. The aim of the present study was to investigate the effects of butylphthalide on cognitive impairment in a streptozotocin-induced diabetic rat model, and the underlying mechanisms of action. A total of 30 healthy male Sprague Dawley rats were randomly divided into the following 2 groups: Normal control $(\mathrm{NC} ; \mathrm{n}=10)$ and diabetes model (DM) groups $(n=20)$. Diabetes was induced in rats in the DM group by intraperitoneal injection of streptozotocin, and these rats were further subdivided into the following 2 groups: Diabetic control $(n=10)$ and butylphthalide-treated groups $(n=10)$. Following 8 consecutive weeks of treatment, a Morris water maze test was performed and the levels of blood fasting plasma glucose (FPG), superoxide dismutase (SOD), malondialdehyde (MDA) and tumor necrosis factor- $\alpha$ (TNF- $\alpha$ ), interleukin (IL)-1 $\beta$, and IL-6 inflammatory cytokines in the hippocampus were measured. FPG levels were significantly decreased in the butylphthalide-treated group when compared with the DM group. In addition, cognitive deficits in diabetic rats were improved following butylphthalide treatment. Furthermore, butylphthalide significantly increased the level of SOD, reduced MDA levels, and reduced TNF- $\alpha$, IL- $1 \beta$, and IL-6 levels in the hippocampus when compared with the DM group. The results of the present study suggest that butylphthalide may be an effective neuroprotective agent to improve cognitive dysfunction during diabetes.
\end{abstract}

Correspondence to: Dr Yuying Zhou, Department of Neurology, Tianjin Huanhu Hospital, 6 Jizhao Road, Jinnan, Tianjin 300350, P.R. China

E-mail: tianzhiyan001@sina.com

Abbreviations: DM, diabetes mellitus; BDNF, brain-derived neurotrophic factor; STZ, streptozotocin; MWM, Morris water maze; FPG, fasting plasma glucose; SOD, superoxide dismutase; MDA, malondialdehyde; TNF- $\alpha$, tumor necrosis factor- $\alpha$; IL-1 $\beta$, interleukin-1 $\beta$; IL-6, interleukin-6

Key words: diabetic rats, cognitive impairment, butylphthalide, superoxide dismutase, brain-derived neurotrophic factor, tumor necrosis factor- $\alpha$, interleukin- $1 \beta$, interleukin- 6

\section{Introduction}

Diabetes is one of the most common metabolic disorders affecting individuals worldwide, and its incidence is increasing each year. Currently, approximately 347 million people are suffering from diabetes mellitus (DM) worldwide and the number will continue to increase (1). The prevalence of diabetes in China has risen significantly in past decades. A large body of research has demonstrated that diabetes is one of the major risk factors for dementia $(2,3)$. Diabetes is associated with alterations in the central nervous system, including cognitive impairment and cerebrovascular disease (4-6). Type 1 diabetes mellitus (T1DM) and type 2 DM (T2DM) have been associated with reduced performance in multiple domains of cognitive function. Previous studies have demonstrated a stronger association between dementia and T2DM compared with T1DM. Specifically, T2DM is associated with a $50 \%$ increase in the risk for dementia, and has been associated with impaired attention, processing and motor speed, executive functioning, and verbal memory. Among the components of metabolic syndrome, hyperglycemia demonstrates the strongest association with the risk of developing cognitive impairment. Hyperglycemia leads to oxidative stress and an inflammatory response, which are the risk factors for Alzheimer's disease in DM. The increased level of oxidative stress under diabetic conditions leads to morphological and functional alterations in different regions of the brain, including the hippocampus and the cerebral cortex (1). In addition, excessive malondialdehyde (MDA) production and decreased efficiency of superoxide dismutase (SOD) in various brain regions have been reported to lead to morphological abnormalities and memory deficits during aging (7). Inflammation is an additional key factor in diabetes-associated cognitive decline. The increased incidence of diabetes is associated with serious socioeconomic problems (1). However, the precise mechanisms underlying diabetes-associated cognitive deficits remain to be elucidated. Therefore, an improved understanding of the mechanisms underlying diabetes-associated cognitive impairment, and the identification of effective treatments for patients with DM is urgently required.

Butylphthalide, a promising drug for the treatment of ischemic stroke, has been approved for clinical use by the State Food and Drug Administration of China $(8,9)$. Preclinical and clinical studies have demonstrated that butylphthalide is an effective neuroprotective agent for the treatment of ischemic stroke (10). In addition, previous studies have investigated the association between butylphthalide and cognition $(11,12)$, as 
well as the use of butylphthalide in the treatment of diabetic rats $(13,14)$. However, the precise mechanisms remain to be elucidated. In the present study, a streptozotocin (STZ)-induced rat model of diabetes was employed to study the possible protective effects of butylphthalide in diabetes-associated cognitive decline.

\section{Materials and methods}

Generation of a diabetic rat model and butylphthalide administration. A total of 30 healthy male Sprague Dawley rats (age, 6 weeks; weight, 180-200 g) were obtained from the Animal Center of Tianjin Huanhu Hospital (Tianjin, China). All experiments were performed in compliance with the regulations approved by the Ethics Committee of Tianjin Huanhu Hospital, and the current study received ethical approval from this committee. Rats were housed in a room at $21-25^{\circ} \mathrm{C}$ with 12 -h light/dark cycles, one rat per cage, and had access to food and water ad libitum. A total of 20 rats received $10 \% \mathrm{STZ}(60 \mathrm{mg} / \mathrm{kg}$ dissolved in citrate buffer, pH 4.5; cat. no. ab142155; Abcam, Cambridge, MA, USA) by intraperitoneal injection. At $72 \mathrm{~h}$ following injection, blood was collected from caudal vein, and blood glucose levels were measured. Diabetic models were considered successful if blood glucose levels were $>16.7 \mathrm{mmol} / 1$. Blood glucose levels were measured weekly, and rats with values $<16.7 \mathrm{mmol} / 1$ were excluded from the study. Diabetic rats were divided into the following 2 groups at random: Diabetic model (DM) control $(n=10)$ and butylphthalide-treated groups $(n=10)$. A total of 10 rats without STZ injection were used as normal controls. Butylphthalide (CSPC NBP Pharmaceutical Co., Ltd., Shijiazhuang, China) was dissolved in vegetable oil. Rats in the butylphthalide-treated group were administered with $80 \mathrm{mg} / \mathrm{kg} /$ day butylphthalide orally for 8 consecutive weeks. The control and DM groups received the same volume of vegetable oil orally.

Fasting plasma glucose (FPG) measurements. FPG was measured in all rats once a week at $24 \mathrm{~h}$ following drug treatment. Tail vein blood was collected for FPG measurements with a blood glucose meter (Accu-Chek; Roche Applied Science, Penzberg, Germany), reading the blood glucose measurement results after $5 \mathrm{sec}$.

Food and water intake measurements. The body weight and water intake of rats were measured once every two weeks for 8 weeks (56 days) immediately after the STZ injection.

Morris water maze (MWM) test. The MWM test (diameter, $50 \mathrm{~cm}$; height, $50 \mathrm{~cm}$; water depth, $40 \mathrm{~cm}$; temperature, $22 \pm 1^{\circ} \mathrm{C}$ ) (14) included a hidden platform (diameter, $10 \mathrm{~cm}$ ) submerged $1 \mathrm{~cm}$ below the surface of the water. On day 1 following the last treatment, all rats were subjected to the MWM test to assess learning and memory abilities. Tests were performed at a fixed time every day for 5 continuous days. A camera located above the center of the maze, together with a tracking system were used to record images and the time taken to locate the platform (the escape latency). If a rat failed to reach the platform within $60 \mathrm{sec}$, it was guided onto the platform (where it remained for $20 \mathrm{sec}$ ), and the latency was recorded as $60 \mathrm{sec}$. A probe trial was performed at day 6 following the final treatment. In this trial, the platform was removed, and rats were allowed to search for the missing platform for $60 \mathrm{sec}$. The frequency of platform crossings was then recorded.

SOD and MDA measurements. The rats in all groups were sacrificed by decapitation at 6 days following the final drug treatment. Hippocampal tissue samples were prepared for SOD and MDA tests. Hippocampal SOD and MDA activity were detected using the Superoxide Dismutase Activity Colorimetric assay kit (ab65354; Abcam). SOD and MDA levels were measured according to the manufacturer's protocol. Absorbance of MDA and SOD was measured using spectrophotometer and microplate reader.

Western blotting analysis of brain-derived neurotrophic factor $(B D N F)$ expression. Hippocampal tissue sections were homogenized with a glass homogenizer in radioimmunoprecipitation assay lysis buffer. The homogenate was centrifuged $\left(4^{\circ} \mathrm{C}, 13,000 \mathrm{x} \mathrm{g}, 10 \mathrm{~min}\right)$ and the supernatants were collected and stored at $-80^{\circ} \mathrm{C}$. The protein concentration was determined using a Bradford protein assay kit (cat. no. ab102535; Abcam). An equal quantity $(100 \mu \mathrm{l})$ of protein from each sample was separated by $12 \%$ SDS-PAGE, and transferred onto a polyvinylidene difluoride membrane by electroblotting. Membranes were blocked for $1 \mathrm{~h}$ at $37^{\circ} \mathrm{C}$ with $5 \%$ non-fat milk, and then incubated overnight at $4^{\circ} \mathrm{C}$ with a rabbit anti-BDNF antibody (1:1,000; Abcam; ab108319) and a rabbit anti- $\beta$-actin (1:2,000; Abcam; ab8227). After several washes in TBST, the membranes were incubated with the appropriate horseradish peroxidase (HRP)-conjugated secondary antibodies (anti-Rabbit IgG H\&L; cat no. ab6271; 1:10,000; Abcam) for $45 \mathrm{~min}$ at $37^{\circ} \mathrm{C}$. LuminataTM Crescendo Western HRP substrate (EMD Millipore, Billerica, MA, USA) was used to visualize the protein bands. Protein bands were detected using the ChemiDoc XRS system (Bio-Rad Laboratories, Inc., Hercules, CA, USA) and Quantity One version 4.62 software (Bio-Rad Laboratories, Inc.).

Enzyme-linked immunosorbent assay (ELISA) analysis of hippocampal cytokine levels. Rat hippocampal tissues were washed and then homogenized on ice with normal saline. Homogenates were centrifuged at $3,000 \mathrm{x}$ g for $10 \mathrm{~min}$ at $4^{\circ} \mathrm{C}$, and the supernatants $(100 \mathrm{ml})$ were used for subsequent analysis. The levels of interleukin (IL)-1 $\beta$, IL-6, tumor necrosis factor- $\alpha$ (TNF- $\alpha$ ) were measured using the Rat TNF- $\alpha$ ELISA kit (cat. no. ab46070, Abcam), the Rat IL-1 $\beta$ ELISA kit (cat. no. ab100768, Abcam) and the Rat IL-6 ELISA kit (cat. no. ab100772; Abcam), respectively, according to manufacturer's protocol. TNF- $\alpha$, IL-1 $\beta$ and IL-6 levels were measured according to the manufacturer's protocol. Absorbance was measured using a microplate reader at a wavelength of $210 \mathrm{~nm}$.

Statistical analysis. The results are presented as the mean \pm standard deviation. All statistical analyses were performed using SPSS (version, 13.0; SPSS, Inc., Chicago, IL, USA). Data were analyzed using repeated measures analysis of variance (ANOVA) followed by least significant difference post hoc analysis. $\mathrm{P}<0.05$ was considered to indicate a statistically significant difference. 


\section{Results}

Butylphthalide decreases FPG levels in diabetic rats. Blood glucose was tested dynamically over the course of 8 weeks. The FPG levels of the DM and butylphthalide-treated diabetic groups were significantly higher than the NC group at all time points $(\mathrm{P}<0.001$; Fig. 1). However, following administration of butylphthalide for 8 weeks, FPG levels were significantly decreased when compared with the DM group $(\mathrm{P}<0.001$; Fig. 1).

Butylphthalide influences body weight and water intake in STZ-induced diabetic rats. A significant reduction in the body weight of STZ-treated rats in the DM group was observed when compared with untreated control rats $(\mathrm{P}<0.01$; Fig. 2A). By contrast, butylphthalide administration significantly reversed the body weight of diabetic rats $(\mathrm{P}<0.05$; Fig. $2 \mathrm{~A})$. The water intake of rats over a $24-\mathrm{h}$ period, which was measured at week 6 following the final drug treatment and when blood glucose levels and body weight had reached a steady state, was increased $\sim 3$-fold in the diabetic rats when compared with the control group, and $\sim 2$-fold compared with the control group (Fig. 2B).

Effects of butylphthalide on cognitive deficits in STZ-induced diabetic rats. The results presented in Fig. 3 demonstrate that butylphthalide significantly ameliorated the cognitive deficits observed in diabetic rats from the DM group. Compared with control group, the escape latency of rats in the DM group was significantly increased $(\mathrm{P}<0.001)$. However, treatment with butylphthalide significantly decreased the escape latency when compared with the DM group $(\mathrm{P}<0.001$; Fig. 3A). In the probe test, the number of platform crossings was significantly decreased in DM group compared with the control group $(\mathrm{P}<0.001$; Fig. 3B). By contrast, treatment with butylphthalide significantly increased the number of platform crossings when compared with the DM group $(\mathrm{P}<0.001$; Fig. 3B).

Effect of butylphthalide on diabetes-induced alterations in oxidative stress. The production of MDA was significantly increased in rats from the DM group when compared with the controls $(\mathrm{P}<0.05$, Fig. $4 \mathrm{~A})$. By contrast, administration of butylphthalide significantly decreased hippocampal MDA levels when compared with the DM group ( $\mathrm{P}<0.05$, Fig. 4A). In addition, SOD levels were significantly reduced in rat hippocampal tissues from the DM group compared with the control group $(\mathrm{P}<0.05$, Fig. 4B). However, treatment with butylphthalide significantly reversed SOD levels in DM rats $(\mathrm{P}<0.05$, Fig. 4B).

Effects of butylphthalide on the protein expression levels of $B D N F$ in the hippocampus of STZ-induced diabetic rats. Following establishment of the STZ-induced rat model of diabetes, a significant decrease in the protein expression levels of BDNF were observed when compared with normal controls $(\mathrm{P}<0.05$, Fig. 5). However, following the administration of butylphthalide for 8 weeks, the protein expression of BDNF in the rat hippocampus was significantly increased when compared to the DM group $(\mathrm{P}<0.05$, Fig. 5).

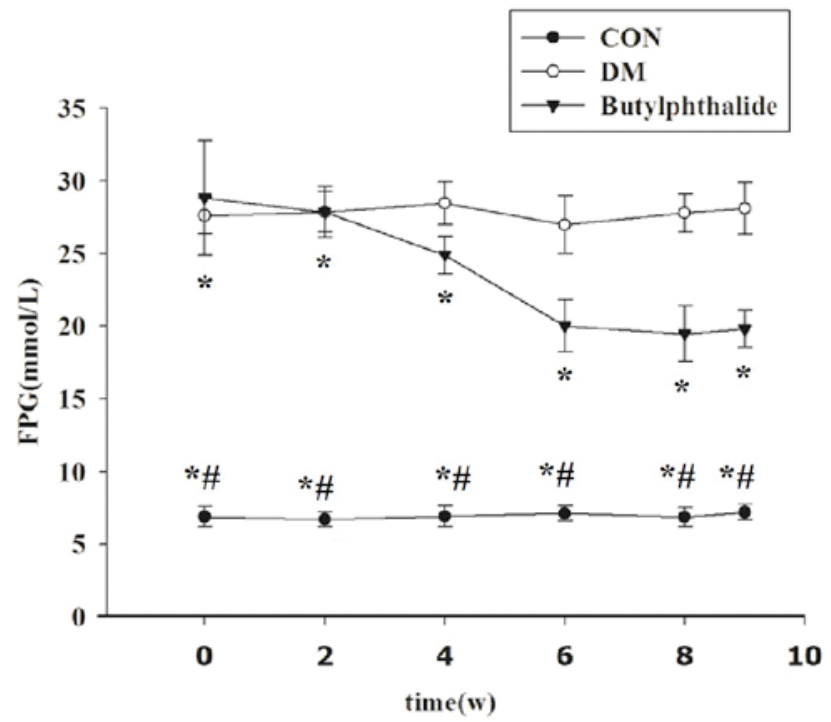

Figure 1. FPG levels in Con, DM and butylphthalide-treated rat groups over the course of 8 weeks. Statistical analysis was performed using repeated measures analysis of variance followed by the least significant difference post hoc test. ${ }^{*} \mathrm{P}<0.05$ vs. $\mathrm{DM}$ group; ${ }^{\#} \mathrm{P}<0.05$ vs. butylphthalide group. $\mathrm{FPG}$, fasting plasma glucose; Con, normal control group; DM, diabetic model group.
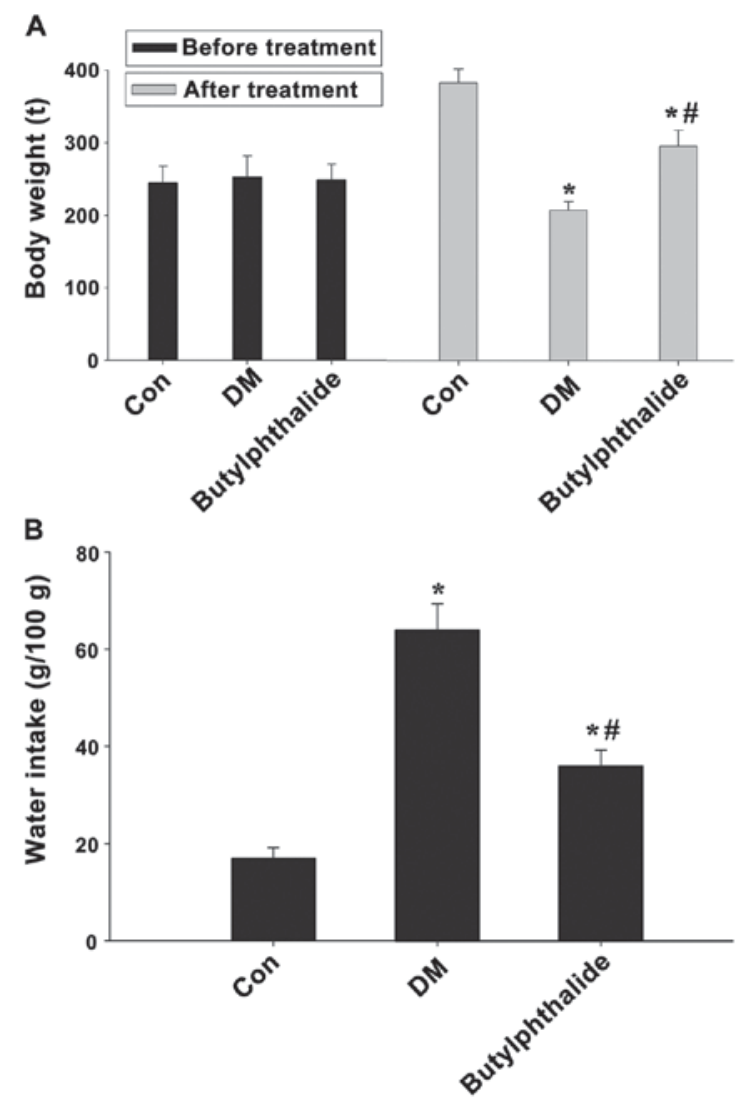

Figure 2. (A) Body weight and (B) water intakes were measured in rats from the Con, DM and butylphthalide-treated groups. Statistical analysis was performed using repeated measures analysis of variance followed by the least significant difference post hoc test. The results are presented as the mean \pm standard deviation $(\mathrm{n}=5)$. ${ }^{*} \mathrm{P}<0.05$ vs. Con group; ${ }^{*} \mathrm{P}<0.05$ vs. $\mathrm{DM}$ group. Con, normal control group; DM, diabetic model group.

Effects of butylphthalide on the level of inflammatory cytokines in the hippocampus of STZ-induced diabetic rats. The 
A
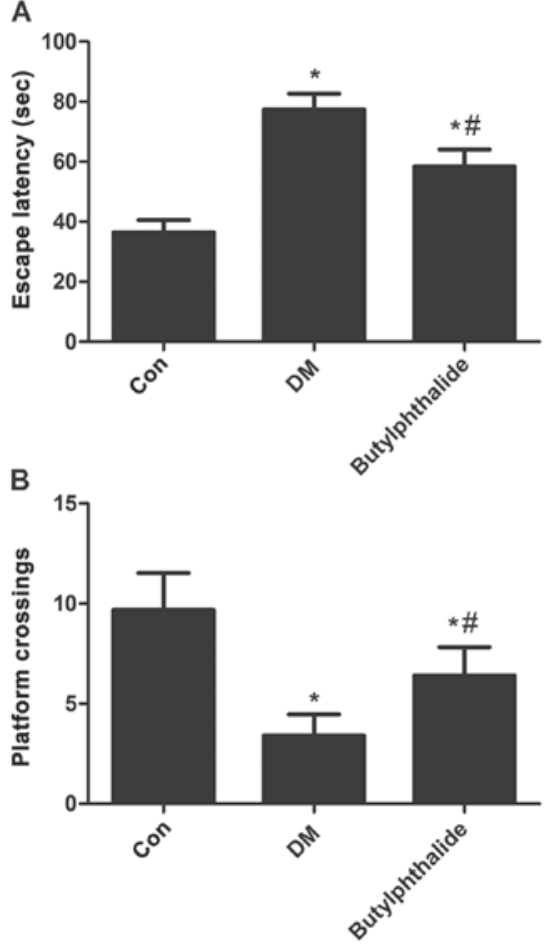

Figure 3. A Morris water maze test was used to analyze the (A) escape latency and (B) number of platform crossings of rats in the Con, DM and butylphthalide-treated groups. The results are presented as the mean \pm standard deviation $(n=5)$. Statistical analysis was performed using repeated measures analysis of variance followed by the least significant difference post hoc test. ${ }^{~} \mathrm{P}<0.05$ vs. Con group; ${ }^{~} \mathrm{P}<0.05$ vs. DM group. Con, normal control group; DM, diabetic model group.
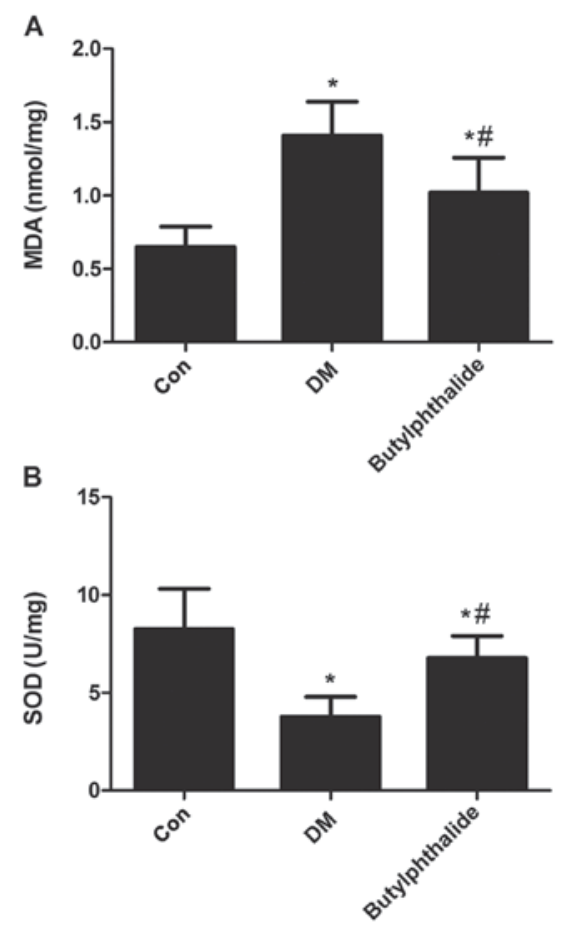

Figure 4. Hippocampal (A) MDA and (B) SOD levels in rats from the Con, DM and butylphthalide-treated groups. Statistical analysis was performed using repeated measures analysis of variance followed by the least significant difference post hoc test. The results are presented as the mean \pm standard deviation $(\mathrm{n}=5)$. ${ }^{*} \mathrm{P}<0.05$ vs. Con group; ${ }^{"} \mathrm{P}<0.05$ vs. DM group. MDA, malondialdehyde; SOD, superoxide dismutase; Con, normal control group; DM, diabetic model group.
A

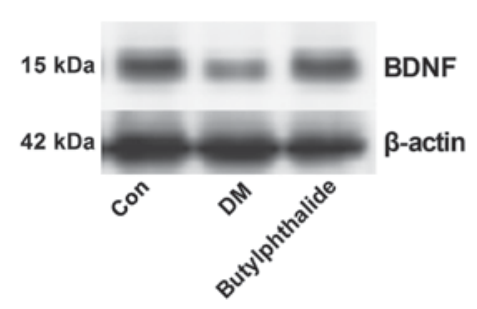

B

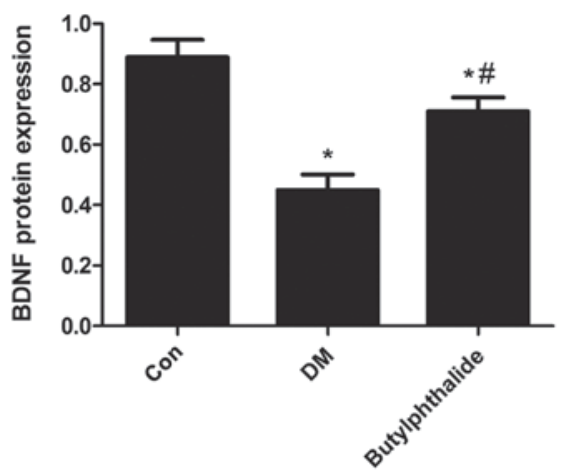

Figure 5. (A) Western blotting analysis of BDNF expression in the hippocampal tissues of rats from the Con, DNA and butylphthalide-treated groups, and (B) quantification of protein band densities. Quantified band densities are presented as the mean \pm standard deviation $(n=5)$. Statistical analysis was performed using repeated measures analysis of variance followed by the least significant difference post hoc test ("P<0.05 vs. Con group; ${ }^{*} \mathrm{P}<0.05$ vs. DM group). BDNF, brain-derived neurotrophic factor; Con, normal control group; DM, diabetic model group.

effects of butylphthalide on the diabetes-associated increase in inflammation were investigated in the present study. As demonstrated in Fig. 6, the expression of inflammatory factors, TNF- $\alpha$, IL-1 $\beta$ and IL-6, were significantly increased in the hippocampus of rats in the DM group when compared with normal controls $(\mathrm{P}<0.01)$. By contrast, butylphthalide treatment significantly reduced the level of these inflammatory factors in the hippocampus of diabetic rats (Fig. 6).

\section{Discussion}

$\mathrm{DM}$ is characterized by dysfunctional insulin secretion and/or insulin function, which leads to the development of metabolic glucose disorders with secondary complications that affect the kidneys, heart, eyes and brain. It has been reported that DM is associated with subtle cognitive decline and an increased risk for reduced cognitive flexibility in the form of dementia. The mechanisms of diabetes-associated cognitive decline include impaired neurogenesis, synaptic dysfunction and reduced blood-brain barrier function (15-18), hyperglycemia and hypoglycemia $(19,20)$, inflammatory and oxidative stress $(15)$, microvascular and macrovascular dysfunction $(21,22)$, and alterations in the insulin signaling pathway (23).

The association between obesity and T2DM has been previously described in an in vivo study, where it was demonstrated that adipose-derived TNF- $\alpha$ levels in rats were elevated with increasing body weight (24). During obesity and metabolic dysfunction, immune cells in adipose tissues secrete proinflammatory cytokines that affect glucose and lipid metabolism (25). The excessive migration of macrophages to adipose tissues and their subsequent activation is a key factor 

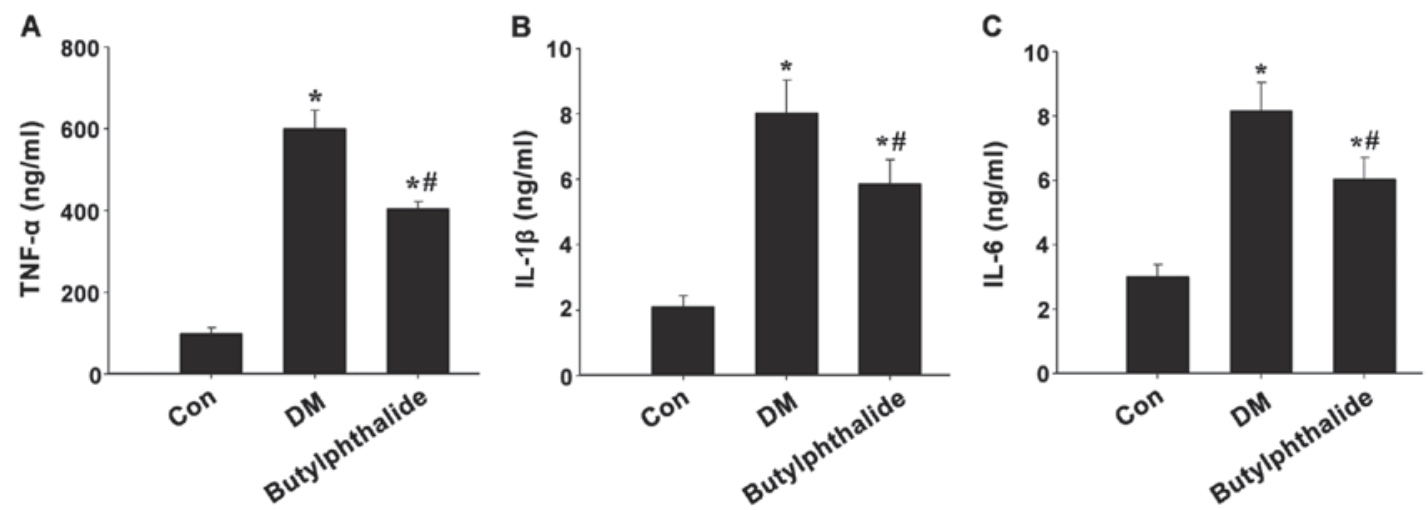

Figure 6. Levels of (A) TNF- $\alpha$, (B) IL-1 $\beta$ and (C) IL-6 levels in the hippocampus of rats from the Con, DM and butylphthalide-treated groups. The results are presented as the mean \pm standard deviation $(n=5)$. Statistical analysis was performed using repeated measures analysis of variance followed by the least significant difference post hoc test. " $\mathrm{P}<0.05$ vs. Con group; ${ }^{\sharp} \mathrm{P}<0.05$ vs. DM group. TNF- $\alpha$, tumor necrosis factor- $\alpha$; IL, interleukin; Con, normal control group; DM, diabetic model group.

for TNF- $\alpha$ and IL-6 production and release, which decreases the activity of the lipoprotein lipase enzyme and increases blood lipid levels.

Oxidative stress has been demonstrated to be involved in the generation of STZ-induced diabetic rats, and has been associated with characteristics of memory decline (26). Due to individual differences, the uptake of STZ is different among rats, therefore the rate of successful generation of a diabetic rat model by oral administration of STZ may be lower when compared with intraperitoneal injection. These methods of model generation will differ in the extent of hyperglycemia and inflammation induced, as well as in the effects of different treatments (27). It is well known that oxidative stress leads to the oxidative damage of biomacromolecules. An increase in MDA is considered to be a specific marker of lipid peroxidation during oxidative damage (28). In addition, oxidative injury may lead to damage of the antioxidant defense system, involving factors such as SOD. Notably, it was previously reported that oxidative stress-induced brain injury contributed to the severe impairment of learning and memory abilities in STZ-induced rats (29). The results of the present study demonstrated that oxidative injury, as evidenced by increased MDA content and decreased SOD activity, and cognitive impairment was observed in a rat model of diabetes. However, these diabetes-associated effects were significantly reversed by butylphthalide treatment.

Butylphthalide is a promising agent for the treatment of ischemic stroke (30). Its mechanisms of action include, decreasing oxidative damage (31), inhibiting platelet aggregation and inflammatory responses $(8,32,33)$, improving mitochondrial function (34) and reducing neuronal apoptosis $(35,36)$. Taking the results of these previous studies into account, there is a strong possibility that butylphthalide may restore cognitive deficits in diabetic rats.

In the present study, diabetic rats underwent continuous butylphthalide treatment for 8 weeks. The escape latency of butylphthalide-treated rats was significantly reduced when compared with untreated diabetic rats, which indicated that STZ-induced cognitive deficits were ameliorated by butylphthalide treatment. In addition, serum FPG levels were significantly decreased in butylphthalide-treated rats, which suggests that butylphthalide may alter glucose metabolism. Hippocampal SOD and BDNF levels were then assessed. SOD is used as a marker of oxidative stress during diabetes $(37,38)$. The results of the present study demonstrated that butylphthalide significantly reversed the diabetes-associated decrease in SOD levels, which suggests that butylphthalide may exhibit antioxidative effects.

BDNF is a type of neurotrophin that serves an important role in regulating the growth and survival of neurons (39). BDNF enhances neuronal plasticity in the hippocampus and improves learning and memory abilities (40). The results of the present study indicated that BDNF protein expression was decreased in STZ-induced diabetic rats, and the expression was upregulated by butylphthalide treatment. These results, together with the known role of BDNF in enhancing neuronal plasticity, suggest that the protective effects of butylphthalide in diabetic rats may involve increased neurogenesis and neuronal plasticity.

Butylphthalide is used for the clinical treatment of acute ischemic stroke, which effectively ameliorates injury of the central nervous system and promotes functional recovery (7). The results of the present study demonstrated that butylphthalide improved diabetes-associated cognitive deficits in rats. It is possible that this may have been due to the antioxidative effects, which is similar to the pharmacological effects of butylphthalide in the treatment of acute ischemic stroke. This was verified by the follow-up experiments, in which butylphthalide is currently used to treat chronic neurodegenerative diseases and those associated with diabetes, butylphthalide may be used to treat chronic neurodegenerative diseases including cognitive impairment in diabetes.

In conclusion, the results of the current study demonstrated that butylphthalide treatment ameliorated the cognitive deficits in STZ-induced diabetic rats. In addition, the protective effects of butylphthalide may be associated with upregulation of BDNF expression and SOD levels in the hippocampus. A single dose of $80 \mathrm{mg} / \mathrm{kg}$ butylphthalide was selected for the purposes of the present study, therefore the effects of different doses need to be investigated in future studies. In addition, clinical studies will be required to validate the neuroprotective effects of butylphthalide on cognitive function in diabetes. 


\section{References}

1. Seto SW, Yang GY, Kiat H, Bensoussan A, Kwan YW and Chang D: Diabetes mellitus, cognitive impairment, and traditional Chinese medicine. Int J Ednocrinol 2015: 810439, 2015.

2. Hölscher C: Diabetes as a risk factor for Alzheimer's disease: Insulin signalling impairment in the brain as an alternative model of Alzheimer's disease. Biochem Soc Trans 39: 891-897, 2011.

3. Katon W, Lyles CR, Parker MM, Karter AJ, Huang ES and Whitmer RA: Association of depression with increased risk of dementia in patients with type 2 diabetes: The diabetes and aging study. Arch Gen Psychiatry 69: 410-417, 2012.

4. Strachan MW, Reynolds RM, Frier BM, Mitchell RJ and Price JF: The relationship between type 2 diabetes and dementia. Br Med Bull 88: 131-146, 2008.

5. Messier C, Awad N and Gagnon M: The relationships between atherosclerosis, heart disease, type 2 diabetes and dementia. Neurol Res 26: 567-572, 2004.

6. Scott AR: Diabetes and Cerebrovascular Disease. In: Vascular Complications of Diabetes: Current Issues in Pathogenesis and Treatment, Second Edition. Donnelly R and Horton E (eds). Blackwell Publishing. Inc, Malden, MA, pp45-50, 2007.

7. Lan Z, Xu X, Xu W, Li J, Liang Z, Zhang X, Lei M and Zhao C: Discovery of 3-n-butyl-2,3-dihydro-1H-isoindol-1-one as a potential anti-ischemic stroke agent. Drug Des Devel Ther 9: 3377-3391, 2015.

8. Peng Y, Xing C, Xu S, Lemere CA, Chen G, Liu B, Wang L, Feng Y and Wang X: L-3-n-butylphthalide improves cognitive impairment induced by intracerebroventricular infusion of amyloid-beta peptide in rats. Eur J Pharmacol 621: 38-45, 2009.

9. Huai Y, Dong Y, Xu J, Meng N, Song C, Li W and Lv P: L-3-n-butylphthalide protects against vascular dementia via activation of the Akt kinase pathway. Neural Regen Res 8: $1733-1742,2013$

10. Xiao S, Kai H, Yang C, Wang X, Ji H, Xu J, Huang Z and Zhang Y: Novel hybrids of 3-n-butylphthalide and edaravone: Design, synthesis and evaluations as potential anti-ischemic stroke agents. Bioorg Med Chem Lett 25: 3535-3540, 2015.

11. Peng Y, Xu S, Chen G, Wang L, Feng Y and Wang X: 1-3-n-Butylphthalide improves cognitive impairment induced by chronic cerebral hypoperfusion in rats. J Pharmacol Exp Ther 321: 902-910, 2007.

12. Wang F, Chen H, Sun XJ and Ke ZJ: Improvement of cognitive deficits in SAMP8 mice by 3-n-butylphthalide. Neurol Res 36: 224-233, 2014

13. Wang F, Ma J, Han F, Guo X, Meng L, Sun Y, Jin C, Duan H, Li H and Peng Y: DL-3-n-butylphthalide delays the onset and progression of diabetic cataract by inhibiting oxidative stress in rat diabetic model. Sci Rep 6: 19396, 2016.

14. Zhang T, Jia W and Sun X: 3-n-Butylphthalide (NBP) reduces apoptosis and enhances vascular endothelial growth factor (VEGF) up-regulation in diabetic rats. Neurol Res 32: 390-396, 2010.

15. Barcia JM, Floresbellver M, Muriach M, Sancho-Pelluz J, Lopez-Malo D, Urdaneta AC, Martinez-Gil N, Atienzar-Aroca S and Romero FJ: Matching diabetes and alcoholism: Oxidative stress, inflammation, and neurogenesis are commonly involved. Mediators Inflamm 2015: 624287, 2015.

16. Wang J, Gong B, Zhao W, Tang C, Varghese M, Nguyen T, Bi W, Bilski A, Begum S, Vempati P, et al: Epigenetic mechanisms linking diabetes and synaptic impairments. Diabetes 63: 645-654, 2014.

17. Huang S, Wang Y, Gan X, Fang D, Zhong C, Wu L, Hu G, Sosunov AA, McKhann GM, Yu H and Yan SS: Drp1-mediated mitochondrial abnormalities link to synaptic injury in diabetes model. Diabetes 64: 1728-1742, 2015.

18. Huber JD: Diabetes, cognitive function, and the blood-brain barrier. Curr Pharm Des 14: 1594-1600, 2008.

19. González-Reyes RE, Aliev G, Ávila-Rodrigues M and Barreto GE: Alterations in glucose metabolism on cognition: A possible link between diabetes and dementia. Curr Pharm Des 22: 812-818, 2016.

20. Perantie DC, Lim A, Wu J, Weaver P, Warren SL, Sadler M, White NH and Hershey T: Effects of prior hypoglycemia and hyperglycemia on cognition in children with type 1 diabetes mellitus. Pediatr Diabetes 9: 87-95, 2008.
21. Krentz AJ, Clough G and Byrne CD: Interactions between microvascular and macrovascular disease in diabetes: Pathophysiology and therapeutic implications. Diabetes Obes Metab 9: 781-791, 2007.

22. Caballero AE, Arora S, Saouaf R, Lim SC, Smakowski P, Park JY, King GL, LoGerfo FW, Horton ES and Veves A: Microvascular and macrovascular reactivity is reduced in subjects at risk for type 2 diabetes. Diabetes 48: 1856-1862, 1999.

23. Sebastião I, Candeias E, Santos MS, de Oliveira CR, Moreira PI and Duarte AI: Insulin as a bridge between type 2 diabetes and Alzheimer disease-how anti-diabetics could be a solution for dementia. Front Endocrinol (Lausanne) 5: 110, 2014.

24. Hotamisligil GS, Shargill NS and Spiegelman BM: Adipose expression of tumor necrosis factor-alpha: Direct role in obesity-linked insulin resistance. Science 259: 87-91, 1993.

25. Tateya S, Kim F and Tamori Y: Recent advances in obesity-induced inflammation and insulin resistance. Front Endocrinol (Lausanne) 4: 93, 2013

26. Wu W, Wang X, Xiang Q, Meng X, Peng Y, Du N, Liu Z, Sun Q, Wang $\mathrm{C}$ and Liu $\mathrm{X}$ : Astaxanthin alleviates brain aging in rats by attenuating oxidative stress and increasing BDNF levels. Food Funct 5: 158-166, 2014

27. Singh R, Kaur N, Kishore L and Gupta GK: Management of diabetic complications: A chemical constituents based approach. J Ethnopharmacol 150: 51-70, 2013.

28. Zhong SZ, Ge QH, Qu R, Li Q and Ma SP: Paeonol attenuates neurotoxicity and ameliorates cognitive impairment induced by d-galactose in ICR mice. J Neurol Sci 277: 58-64, 2009

29. Zhao CH, Liu HQ, Cao R, Ji AL, Zhang L, Wang F and Yang RH: Effects of dietary fish oil on learning function and apoptosis of hippocampal pyramidal neurons in streptozotocin-diabetic rats. Brain Res 1457: 33-43, 2012.

30. Wang X, Wang L, Li T, Huang Z, Lai Y, Ji H, Wan X, Xu J, Tian J and Zhang Y: Novel hybrids of optically active ring-opened 3-n-butylphthalide derivative and isosorbide as potential anti-ischemic stroke agents. J Med Chem 56: 3078-3089, 2013.

31. Huang JZ, Chen YZ, Su M, Zheng HF, Yang YP, Chen J and Liu CF: dl-3-n-Butylphthalide prevents oxidative damage and reduces mitochondrial dysfunction in an MPP(+)-induced cellular model of Parkinson's disease. Neurosci Lett 475: 89-94, 2010.

32. Chen M, Qi L, Min T, Wen S, Pi R and Lin D: Synthesis and biological evaluation of n-butylphthalide derivatives as anti-platelet aggregation agents. Nat Prod Res: 1-4, 2016 (Epub ahead of print).

33. Wang HM, Zhang T, Huang JK and Sun XJ: 3-N-butylphthalide (NBP) attenuates the amyloid- $\beta$-induced inflammatory responses in cultured astrocytes via the nuclear factor- $\mathrm{\kappa B}$ signaling pathway. Cell Physiol Biochem 32: 235-242, 2013.

34. Wang YG, Li Y, Wang CY, Ai JW, Dong XY, Huang HY, Feng ZY, Pan YM, Lin Y, Wang BX and Yao LL: L-3-n-butylphthalide protects rats' cardiomyocytes from ischaemia/reperfusion-induced apoptosis by affecting the mitochondrial apoptosis pathway. Acta Physiol (Oxf) 210: 524-533, 2014.

35. Yin W, Lan L, Huang Z, Ji J, Fang J, Wang X, Ji H, Peng S, $\mathrm{Xu} \mathrm{J}$ and Zhang Y: Discovery of a ring-opened derivative of 3-n-butylphthalide bearing NO/H2S-donating moieties as a potential anti-ischemic stroke agent. Eur J Med Chem 115: 369-380, 2016.

36. Li J, Yin L, Ogle M, Xin Zhou, Minke Song, Shan Ping Yu and Ling Wei: dl-3-n-Butylphthalide prevents neuronal cell death after focal cerebral ischemia in mice via the JNK pathway. Brain Res 1359: 216-226, 2010.

37. Tiwari BK, Pandey KB, Abidi AB and Rizvi SI: Markers of oxidative stress during diabetes mellitus. J Biomark 2013: 1-8, 2013.

38. Bandeira SM, Guedes Gda S, da Fonseca LJ, Pires AS, Gelain DP, Moreira JC, Rabelo LA, Vasconcelos SM and Goulart MO: Characterization of blood oxidative stress in type 2 diabetes mellitus patients: Increase in lipid peroxidation and SOD activity. Oxid Med Cell Longev 2012: 819310, 2012.

39. Pencea V, Bingaman KD, Wiegand SJ and Luskin MB: Infusion of brain-derived neurotrophic factor into the lateral ventricle of the adult rat leads to new neurons in the parenchyma of the striatum, septum, thalamus, and hypothalamus. J Neurosci 21: 6706-6717, 2001.

40. Mizuno M, Yamada K, Olariu A, Nawa H and Nabeshima T: Involvement of brain-derived neurotrophic factor in spatial memory formation and maintenance in a radial arm maze test in rats. J Neurosci 20: 7116-7121, 2000. 